\title{
Shooting sports as the means of professional-applied physical training at maritime University
}

Igor Zub*

Admiral Makarov State University of Maritime and Inland Shipping, 198035, Saint Petersburg, Russia

\begin{abstract}
Training specialists of water transport at maritime University presents a complex of knowledge and skills. They are necessary for the specialists in order to fulfill their professional duties. Apart from special subjects an important stage of training becomes professional-applied physical training. Its complexes are formed from different kinds of sport or during going in for professional-applied kinds of sport and provide professionally important qualities development, which define during professiograms creation according to each specialty. During water transport specialists' professiograms analysis it is seen that the general professionally important qualities are vestibular apparatus steadiness and statokinetic resistance. These qualities help specialists of water transport realize their job duties during pitching.

One of the kinds of sport, the effectiveness of which depends on statokinetic resistance, is shooting sports. Shooting sports is a separate kind of sport, but as a kind of sport it is included into the complex of marine all-round competitions. Marine all-round competitions are professional-applied kind of sport for the specialists of water transport. They provide necessary professionally important qualities development. Shooting sports is the means of the following things development: statokinetic resistance, the ability to focus attention, psycho-emotional resistance. It is important during extreme situations. The conclusion, received as a result of scientific works analysis, helps to define the place of this kind of sport in the training diagram of marine all-round athletes, for getting the effect of positive transfers in the training process.
\end{abstract}

\section{Introduction}

The activity of water transport specialists is realized in conditions, which can be called extreme. Extreme conditions of work on a vessel can be divided into two groups - not depending on a person (storm, low visibility, humidity, low or high temperature) and extreme conditions, created because of the mistakes of a person. The latter depend both on professional training of specialists and on his psycho-physical qualities. In such situations it is important not only to have psychic professionally important qualities (PIQ), but also physical ones.

Professional training of water transport specialist includes not only teaching special knowledge and practical training according to the specialty, but also professional-applied physical training (PAPT). PAPT is one of the aspects of a complex training among cadets of maritime University studying in swimming specialties. PAPT includes kinds of sport and their elements. They develop PIQ of specialists of water transport. One of the kinds of sport, which develop PIQ is marine all-round competitions, which includes the following: rowing and sailing regatta, running, swimming and shooting.

For all water transport specialist general PIQ, regardless of their specialty, would be statokinetic resistance and seasickness resistance. Seasickness resistance is achieved by means of vestibular apparatus development. Statokinetic resistance is connected with vestibular apparatus and reflects a person's resistance to stimuli influence during body position change. It is demonstrated in terms of pitching. For statokinetic resistance and vestibular apparatus development the exercises from different kinds of sport were used [1,2]. Psycho-emotional state of a person also influences statokinetic resistance. Emotionally steady people have the ability to mobilize their psychic and physical abilities in case of extreme situation. In case of such situations it is important for water transport specialists to preserve working capacity and psycho-emotional balance. These skills are achieved by means of systematic training lessons of professional skills and physical and psychic qualities development.

Shooting sports, which is included into the complex of marine all-round competitions, belongs to acyclic, difficult coordinating kinds of sport, develops both physical and psychic qualities of a person. In this kind of sport result mainly depends on emotional state of an athlete. Shooting sports (further shooting), develops statokinetic resistance of a person and his vestibular apparatus, forming "seasickness" resistance of an organism [2].

\section{Literature review}

\footnotetext{
* Corresponding author: zubiv@mail.ru
} 
Motor activity of a person is a difficult process. It is conditioned by the ability to regulate the poses and body positions for coordination of movements achievement. The activity of a person, both professional and sports activity, is conditioned by the ability to preserve effectively the definite statokinetic and statodynamic poses [3, 4, 5], in terms of minimal muscle efforts [6].

Vertical position of a person is considered as a private case of movement. In this position a person preserves the pose owing to static steadiness. It is determined as the ability of a person to resist any balance violation. Micro movements, which happen during vertical pose preservation, are connected with the frequency of heart rate, hemodynamics, breathing [7]. The reaction of cardiovascular system to vestibular signal influences vestibular resistance. In case of static support of body balance, eyesight has a great influence on movements control. The impulses from visual analyzer activate the muscles. They take part in supporting postural control over static body steadiness. In case of systematic sport lessons, as a result of interaction between visual, proprioceptive, vestibular sensory systems, the effectiveness of statokinetic resistance support increases. Statokinetic resistance formation depends both on vestibular apparatus and on interaction between sensory systems [4, 8].

If a person was influenced by short-term external powers, which exceeded powers of resistance, and after they finish their influence a person returns to the initial balanced position. Such ability of a person is called a dynamic resistance [7].

Motor activity of athletes, who go in for shooting sports, happens in terms of standard static poses. Physical tiredness, caused by long-term static loads, leads to static resistance and the ability to preserve balance violation [8, 9 , 10]. The function of balance violation among athletes can decrease in terms of shoulder girdle muscles tiredness [11].

A shooter's pose preservation during the exercise fulfillment is provided by statokinetic resistance, static endurance and the sense of balance. Micro movements coordination during shooting provides attention focusing on body parts positions in terms of the support during the all phases of a shot $[12,13,14]$. The ability to control the stand while shooting forms athlete's complex motor image from ready position till the shot fulfillment, with attention focusing on the parts of the body position relative to the area of the support [12]. Statokinetic resistance of "a shooter- weapontarget" system support is realized owing to the regulated legs movements $[9,12,15,16]$. During aiming vertical pose support there is passive knee joints "closure". It leads to the degrees of freedom decrease in the system of a person's body. At the same time, statokinetic resistance support happens not owing to controlling influences of some joints, but owing to intermuscular interactions. They are individual for each athlete [17].

Technical training in shooting provides sense of space, muscle-joint sense and sense of time development. They provide the rhythmicity of the fulfilled actions [18]. During the result of shooting analysis the information influences vestibular apparatus. It influences intermuscular coordination of body, legs muscles, arms and forearm muscles developing and improving muscle-joint and spatial perception, provides interaction between vestibular and visual analyzers and it has a positive influence on statokinetic resistance development. In ready and aiming position an athlete experiences static loads, during which breath holding happens and energy supply happens in anaerobic regimen owing to creatine phosphate mechanism. Breath holding helps to fulfill an effective shot. It is explained by the fact that breath holding increases the activity of respiratory muscles and it leads to spinal column stiffness increase and pose steadiness [19].

Great volume of the training load is fulfilled in terms of static tensions, which can cause hypoxic state. It is connected with the fact that during static loads oxygen provision of an organism is lower, than during dynamic loads $[13,20,21]$. These factors lead to physiological changes in an athlete's organism. They depend on the fulfilled during the training load. Appearing in this case different in tension hypoxic states are the result of nonconformity between energetic needs of an organism and opportunities of their satisfaction [22].

Static loads lead to blood circulation of lower extremities violation. It leads to general working capacity and the effectiveness of the exercise fulfillment decrease [23]. The results of cardiovascular system diagnostics can show general and special physical readiness of an athlete [24], and estimate his adaptive potential [25]. Motor functions of an athlete equally depend on the degree of his physical and psychological qualities and states. Psycho-emotional tension, which appears, can change the character of an athlete's behavior both in terms of the training and competitive activity $[26,27]$. If in another kinds of sport psycho-emotional tension can be demonstrated in a form of aggression or aggressive attitude to the opponent, in shooting sport the opponent of an athlete is an athlete himself [28]. For any athlete it is important to focus attention both on inner and external form of activity [28, 29, 30]. The ability to focus attention decreases the level of psycho-emotional tension, an athlete gains the state of psychic readiness or psychoemotional steadiness for competitive activity realization. It can be considered the activity in extreme conditions [30].

Psycho-emotional steadiness development helps to mobilize psycho-somatic reserves of an organism It is demonstrated in the ability to resist competitive stresses. Each athlete has its own level of psycho-emotional steadiness. It depends on personal competitive experience and is demonstrated in behavior individualization in different situations [31]. At the same time, constantly growing load, previous competitions analysis can lead to anxiety state appearance. Regardless of the level of anxiety this state has a negative influence on an athlete's activity. Teaching athletes the techniques of relaxation and self-control provide the level of psycho-emotional steadiness increase [32].

\section{Materials and Methods}

The following methodology of the research was used in the work: scientific sources analysis. Scientific sources analysis was held in order to search for and summarize modern methods of training in shooting sports concerning physical 
(statokinetic resistance) and psychological (emotional steadiness) qualities development with further transfer of these methodologies for PIQ development among the future specialists of water transport by means of marine all-round competitions.

\section{Results and Discussion}

During the technique of shooting mastering and static balance purposeful upbringing steadiness of "shooter-weapontarget" systems is achieved. Steadiness is conditioned by coordinating abilities of a shooter and a long-term vertical position holding is realized owing to muscles tension. During aiming a shooter holds his breath. It leads to lungs capacity decrease and static efforts in case of pose and a weapon holding, lead to blood circulation violation. In order to compensate negative influences of static loads on an organism lessons in a shooting gallery should be alternated with such kinds of sport as running, swimming, rowing [13, 21]. As shooting is one of the kinds of marine all-round competitions, it is necessary to take into account the transfers while planning shooting training process. The training loads according to all-round competitions and their alternation should give a positive transfer of one kind into another one [33].

During the training lessons athletes' immunity to a disturbance is formed. The ability to control own emotions and preserve tranquility in extreme situations transfers to everyday and professional activity. As it is mentioned in the work [34] the main external extreme factors for an athlete are the following: new competitive environment conditions, unexpected situations, which appear during the competitions, time limit of a competitive exercise, technical characteristics of a weapon.

Inner factors are conditioned by psycho-emotional steadiness of an athlete, his emotional state, physical loads. In order to regulate psycho-emotional balance psychological training is held. It includes different ways of psychoemotional balance preservation or auto-training. The problem of resistance to stress, as PIQ, is usual not only for shipboard personnel, but also for the specialists- dock workers. Shipboard personnel and dock workers have the specificity of work, which can be characterized as a monotonous and situational, quickly changing activity. The specialist, who doesn't have resistance to stress, can show not adequate reaction in this case. It can lead to emergency situation. While teaching by PAPT not only physical, but also psychological qualities are mastered among the athletes.

Navigator experiences static loads identical with the loads of an athlete-shooter when the sea is calm. During pitching, apart from static resistance, water transport specialist has to be able to coordinate the center of gravity in terms of the support area or in other words, should be able to preserve static-dynamic resistance. It is developed with the help of special exercises. In case of well-developed static-dynamic resistance and general static endurance, if vestibular apparatus is not developed, a ship specialist would have difficulties with pitching resistance. It can lead to emergency situation. That is why vestibular apparatus development is the main aim of PAPT.

In accordance with the International Convention "On training and certificating sailors and keeping the watch" a shipboard personnel should correspond with several demands concerning physical training. They are necessary both for keeping the watch and vital activity of a vessel provision and for acting in extreme situations [35].

Behavior in extreme situations depends on the characteristics of a personality, how a person perceives a situation. Reaction to the extreme situation can be different: panic, adaptation, positive traits of character demonstration, the ability to control the situation. The latter reaction causes positive shifts in psychological development of an individual, gives an opportunity to realize further development in professional sphere. Psycho-emotional steadiness, which is developed during shooting lessons, has a positive transfer to professional activity. Intermuscular coordination, developed during shooting lessons, is necessary for the specialists of marine engineering service during equipment repair and regulation [1].

Taking into account the fact that during breath holding an organism experiences lack of oxygen (hypoxic state), it becomes obvious that during shooting lessons it is necessary to pay attention to general physical readiness. It is based on cyclic kinds of sport for respiratory and cardiovascular systems development. If we consider shooting sports as one of the kinds of marine all-round competitions, we have to answer the following question: "How to plan the trainings in order to get the effect of positive transfers?" It depends on planning the training lessons of other kinds of a complex. In training lessons of rowers we use static-dynamic exercises [36]. That is why the training lessons of these kinds shouldn't be combined or planned sequentially.

Shooting sports develops such PIQ, which are necessary for water transport specialists: statokinetic resistance, intermuscular coordination for balance holding, manual dexterity. These qualities are demonstrated during repair works organized by the specialists in terms of sea pitching and in case of static pose holding. Regulating works fulfillment demands attention concentrating and psycho-emotional; steadiness, especially if these works are held in extreme conditions (for example, main engine failure during the storm).

The work of shipboard personnel happens in conditions of not changing environment. It also influences a person's psyche. During shooting lessons an athlete fulfills 180-240 shots. These training lessons have monotonous character, but at the same time, they develop such qualities as attention focusing on inner and external factors for the set aim achievement.

\section{Conclusions}


As a result of systematic training lessons athlete's regulating mechanisms steadiness increases. These mechanisms provide balance and statokinetic resistance support. It is provided by interaction between vestibular apparatus, proprioceptive and visual analyzers and central nervous system.

Control over external and inner factors, attention focusing on competitive exercise fulfillment helps to master psycho-emotional steadiness in terms of competitive activity. It for athletes is equal to extreme situation. Psychoemotional steadiness, mastered during the training lessons, transfers to everyday and professional activity. It is important for water transport specialists, as the specificity of marine activity refers to ex treme one.

During the training lessons, which are organized in static regimen and breath holding, lower extremities blood circulation violates. It leads to working capacity decrease. For physical fitness support athletes-shooters are recommended to go in for cyclic kinds of sport. The complex of marine all-round competitions includes such cyclic kinds as running, swimming and rowing. Shooting trainings are mainly held during winter period, when there is no opportunity to sail. Sailing and boating influence vestibular apparatus development. It helps to increase statokinetic resistance. Training swimming and running improve working capacity of legs muscles. It has positive influence on balance and statokinetic resistance support.

\section{References}

1. I.V. Zub, Collection of scientific articles of the national scientific-practical conference of teaching staff Federal State Budgetary Educational Establishment of Higher Education "Admiral Makarov State University of Maritime and Inland Shipping" (publishing house Admiral Makarov State University of Maritime and Inland Shipping, Saint-Petersburg, 1, 202-213, 2018)

2. I.V. Zub, F.V. Korol, Strategic directions of University system of physical culture reformation: collection of scientific works of All-Russian scientific-practical conference with the International participation (publishing house of Polytechnic University, Saint-Petersburg, 346-349, 2018)

3. T.F. Abramova, V.V. Arkov, V.V. Ivanov, T.M. Nikitina, D. Suprun, Bulletin of sports science, 4, $64-69$ (2008)

4. A.A. Udalova, Scientific notes of P.F. Lesgatf University, 3(121), 161-166 (2015)

5. V. Yagello, A. Zyulkovska, Pedagogics, psychology and medical-biological problems of upbringing, 12, 141-145 (2008)

6. V.S. Kozhevnikov, Bulletin of Izhevsk State Technical University named after M.T. Kalashnikov, 20(2), 165-167 (2017)

7. V. Boloban, Yu. Litvinenko, T. Nizhnikovski, Science in the Olympic sport, 1, 27-35 (2012)

8. A.S. Nazarenko, A.S. Chinkin, Science and sport: modern tendencies, 7(2), 78-85 (2015)

9. A.S. Nazarenko, F.A. Mavliev, N.V. Rylova, A.S. Chinkin, Practical medicine, 3(88), 65-68 (2015)

10. R.Yu. Nikolaev, A.A. Melnikov, A.V. Borisov, Proceedings of Southern Federal University. Technical sciences (Medical information systems), 10(159), 227-236 (2014)

11. A.S. Nazarenko, F.A. Mavliev, N.Sh. Khasnutdinov, Scientific notes of P.F. Lesgaft University, 5(123), 135-138 (2015)

12. V.V. Shilenko, G.A. Kuzmenko, Bulletin of sports science, 2, 24-30 (2017)

13. T.P. Afinogenov, B.A. Nazarenko, Society: sociology, psychology, pedagogics, 1, 87-89 (2016)

14. V.G. Nyrkov, Bulletin of Ufa juridical Institute of the Ministry of internal affairs of Russia, 3, 49-52 (2013)

15. G.N. Germanov, I.A. Sabirova, A.V. Chernykh, S.V. Serdochenko, Scientific notes of P.F. Lesgaft University, 5, 36-40 (2014)

16. V.V. Khramov, E.O. Shirshova, E.L. Matova, Person. Sport. Medicine, 19(S1), 99-105 (2019)

17. E.A. Lukunina Candidate's thesis (Russian State Academy of Physical Culture, Moscow)

18. V.A. Pogodin, Bulletin of sports science, 1, 62-65 (2016)

19. R.Yu. Nikolaev, A.A. Melnikov, Bulletin of Northern (arctic) Federal University. Series: medical-biological sciences, 4, 58-65 (2014)

20. L.V. Tarasova, E.R. Yashina, T.F. Abramova, Bulletin of sports science, 5, 37-42 (2017)

21. V.S. Simonenkov, S.R. Gilazieva, N.S. Shumilina, Bulletin of Orenburg State University, 2(202), 135-140 (2017)

22. E.L. Bakhchevan, S.V. Chebotar, Bulletin of ONU. Biology, 20(1(36)), 69-81 (2015)

23. L.V. Safonov, Yu.I. Sternin, V.L. Sfonov, Bulletin of sports science, 5, 34-39 (2015)

24. L.V. Tarasova, E.R. Yashina, T.F. Abramova, Bulletin of sports science, 6, 45-48 (2017)

25. N.P. Garganeeva, I.F. Taminova, L.I. Tyukalova, I.N. Vorozhtsova, I.L. Bukhovets, E.N. Pashkova, Cardiovascular therapy and prophylaxis, 14(5), 60-66 (2015)

26. D.S. Sharipova, Bulletin of Perm University. Philosophy. Psychology. Sociology, 4(20), 71-77 (2014)

27. I.A. Sabirova, Scientific notes of P.F. Lesgaft University, 5(63), 93-97 (2010)

28. A.Yu. Sharapov, S.D. Zubrilkin, S.C. Yakimov, Scientific-practical electronic journal "Alley of Science"[Alleya Nauki], 13 (2017) URL: http: Alley-science.ru

29. A.V. Chaban, Z.M. Kuznetsova, A.V. Ryabchuk, Pedagogical-psychological and medical-biological problems of physical culture and sport, 12(2), 146-153 (2017)

30. E.Yu. Korobeynikova, S.V. Leonov, I.S. Polikanova, National psychological periodical, 2(26), 35-45 (2017)

31. P.V. Rodichkin, F.F. Kostov, G.V. Buznik, Theory and practice of physical culture, 10, 39-42 (2015)

32. F.F. Kostov, P.V. Rodichkin, G.V. Buznik, Theory and practice of physical culture, 8, 56-58 (2016) 
33. I.V. Zub, V.A. Kurys, A.Zh. Berekenov, Physical culture of students: Materials of All-Russian scientific-practical conference. Saint-Petersburg, June, 13, 2019 (POLYTECH-PRESS, Saint-Petersburg, 308-311, 2019)

34. L.V. Tarasova, Bulletin of sports science, 3, 25-27 (2009)

35. International Convention on training and certificating sailors and keeping the watch. (adopted on the assembly of IMKO, July, 7, 1978) URL: http://us.msun.ru/us/document/STCW_Rus.pdf

36. I.V. Zub, A.V. Bykov, V.A. Kurys, Proceedings of the First International Volga Region Conference on Economics, Humanities and Sports (FICEHS 2019), 114, 746-749 (2019) 